\title{
Qualitative Analysis of Both Hyperbolic and Non-hyperbolic Equilibria of a SIRS Model with Logistic Growth Rate of Susceptibles and Inhibitory Effect in the Infection
}

\author{
Jayanta Kumar Ghosh ${ }^{1}$, Uttam Ghosh ${ }^{2}$, Susmita Sarkar ${ }^{2}$ \\ ${ }^{1}$ Boalia Junior High School, Nadia, West Bengal, India \\ E-mail: jayantaghosh.326@rediffmail.com \\ ${ }^{2}$ Department of Applied Mathematics, University of Calcutta, Kolkata, India \\ E-mail:uttam_math@yahoo.co.in,susmita62@yahoo.co.in
}

Received: 20 May 2018; revised: 19 December 2018; accepted: 20 December 2018; published online: 31 December 2018

\begin{abstract}
This paper describes a SIRS model with the logistic growth rate of susceptible class. The effect of an inhibitory factor in the infection is also taken into consideration. We have analysed local as well as global stabilities of the equilibrium points (both hyperbolic and non-hyperbolic) of the system and investigated the Transcritical bifurcation at the disease free equilibrium point with respect to the inhibitory factor. The occurrence of Hopf bifurcation of the system is examined and it was observed that this Hopf bifurcation is either supercritical or subcritical depending on parameters. Some numerical simulations are carried out for the validity of theoretical results.

Key words: inhibitory factors, logistic growth, losses immunity, global stability, hopf bifurcation, transcritical bifurcation, centre manifold theory
\end{abstract}

\section{INTRODUCTION}

The dynamics of infectious diseases is an important research branch in mathematical epidemiology. Mathematical modelling in epidemiology provides understanding of the underlying mechanisms that influence the spread of infectious diseases and suggests its control strategies. One of the early triumphs of mathematical epidemiology was the formulation of a simple model by Kermack and McKendrick (1927) [1]. More developments and progresses have been particularly made during the past three decades. Massive mathematical models have been formulated to study the dynamical behaviour of various infectious diseases which shows rich nonlinear phenomena [2-6].

Researchers formulate compartmental models based on assumptions on the rates of flow between different classes of members of the population $[3,7,8]$. To formulate the compartmental model two important factors play the crucial role: one is the growth rate of susceptible class and the other is the rate of infection [9-19]. The authors usually consider the constant growth rate [17], exponential growth rate and logistic growth rate $[9-12,26]$. The logistic growth rate is considered in those models where food supply, space capacity or carrying capacities of the system are limited. On the other hand, the authors consider a different type of the incidence rate, i.e. the infection rate of susceptible individuals through their contacts with infected individuals. The first one is the bilinear incidence rate $\beta S I[9,10]$, where $S$ and $I$ are, respectively, the number of susceptible and infected individuals in the population and $\beta$ is the transmission rate of infection. The other is the saturated incidence rate of the form $\frac{\beta S I}{1+\alpha S}$ or $\frac{\beta S I}{1+\alpha I}[12,15,17]$. In the incidence rate $\frac{\beta S I}{1+\alpha S}, \alpha$ is defined as inhibition coefficient 
and this incidence rate increases as the susceptible population increases and ultimately it tends to $\beta I / \alpha$ as $S \rightarrow \infty$ if $I$ is finite. This type of infection is sometimes named as 'incidence rate with psychological effect', because the effect of $\alpha$ stems from epidemic control (taking appropriate preventive measures and awareness) and the rate of infection decreases as the inhibitory coefficient $\alpha$ increases.

To study disease dynamics of the SIR epidemic model, Enatsu et al in [9] and Wang et al in [10] considered the growth rate of the susceptible class as the logistic type and the rate of infection as bilinear type mass action. Wang et al in [11] studied the SIR model with the logistic type growth rate of susceptible class with the saturated type treatment rate. Kar and Mandal in [12] considered the SIR epidemic model with the logistic type growth rate of susceptible populations with the saturated type $\frac{\beta S I}{1+\alpha S}$ infection rate.

In this paper, we have considered a SIRS model with the logistic type growth rate of susceptible population and the rate of infection is affected by the inhibitory effect. We have also considered in our model that some recovered individuals lose immunity, [25], and so they become susceptible. SIRS models represent a class of airborne diseases, for example seasonal influenza, but in this paper we focus on a generic SIRS model. This model is the extension of the model considered in [12]. The main objective of this paper is to discuss the stability or instability of the both hyperbolic and non-hyperbolic equilibrium points and exhibition of Transcritical and Hopf bifurcation. The stability analysis of non-hyperbolic equilibrium points will be investigated here by using the Centre Manifold Theory and we will also analyse the role of awareness or inhibitory factors to control the infection of infectious diseases. The paper is organized as follows. In section 2 we have formulated the model, while in section 3 we discuss the existence of equilibria and we have obtained the basic reproduction number $R_{0}$. Section 4 is devoted for local and global stability of equilibrium points, and in section 5 we have shown that the system experiences a Transcritical and Hopf bifurcation at the equilibrium points. Finally, a theoretical finding is justified using the numerical simulation in section 6 and boundedness and permanence of the system are shown in the appendix.

\section{THE BASIC MATHEMATICAL MODEL}

Here we assume that the susceptible class follows the logistic growth rate and the incidence rate is of saturated type that reflects the "psychological effect" or "inhibition effect" [20]. In the proposed model we also consider the loss of immunity of recovered class. Let $S(t), I(t)$ and $R(t)$ be the number of susceptible, infected and recovered individuals at time $t$, respectively. Incorporating all the assumptions described above, the governing differential equations of the model can be written as

$$
\begin{aligned}
& \frac{d S}{d t}=r S\left(1-\frac{S}{k}\right)-\frac{\beta S I}{1+\alpha S}-d S+\mu R, \\
& \frac{d I}{d t}=\frac{\beta S I}{1+\alpha S}-(d+\gamma) I \\
& \frac{d R}{d t}=\gamma I-(d+\mu) R
\end{aligned}
$$

with nonnegative initial conditions. Parameters used in system (1) are nonnegative and listed in Table 1.

Since the exact solution of the nonlinear autonomous system (1) is impossible to find, we are analysing the qualitative behaviour of the solutions in the neighbourhood of the equilibrium points.

\section{EXISTENCE OF EQUILIBRIA AND THE BASIC REPRODUCTION NUMBER}

The system (1) has always trivial equilibrium point $E_{0}(0,0,0)$. The axial equilibrium $E_{1}\left(S_{1}, 0,0\right)$ exists only when $r>d$. The system has only one endemic equilibrium point $E_{2}\left(S_{2}, I_{2}, R_{2}\right)$ if the thresholds: $\Delta_{1}>1$ and $\Delta_{2}>1$, where

$$
\begin{aligned}
& S_{1}=k\left(1-\frac{d}{r}\right) \\
& S_{2}=\frac{1}{\alpha\left(\Delta_{1}-1\right)}, \\
& I_{2}=\frac{(d+\mu)\left(d+\frac{r S_{2}}{k}\right) S_{2}\left(\Delta_{2}-1\right)}{d(d+\mu+\gamma)}, \\
& R_{2}=\frac{\gamma I_{2}}{d+\mu}, \\
& \Delta_{1}=\frac{\beta}{\alpha(d+\gamma)} \text { and } \\
& \Delta_{2}=\frac{k \alpha r\left(\Delta_{1}-1\right)}{k \alpha d\left(\Delta_{1}-1\right)+r} .
\end{aligned}
$$

So the disease free equilibrium point (DFE) $E_{1}$ will exist if the intrinsic growth rate is greater than the natural death rate. Again, from the expression of $\Delta_{1}$ it is clear that $\Delta_{1}$ will be large if $\beta$ is high or $\alpha$ is low. Thus the rate of infection $(\beta)$ and inhibitory parameter $(\alpha)$ play an important role for the existence of $S_{2}$ and $I_{2}$. From the expression of $I_{2}$ it is clear that with the increase of $\Delta_{2}$ (when $\beta$ increases or $\alpha$ decreases) $I_{2}$ will increase, that is, the number of the infected will increase. Thus, to control the disease we have to increase inhibitory coefficient $\alpha$.

Since the considered model has a DFE at which the population remains in the absence of disease, the model has a threshold parameter known as the basic reproduction number $R_{0}$ which plays an important role to control the disease. 
Tab. 1. Model parameters and their descriptions

\begin{tabular}{c|c}
\hline Parameters & Interpretations \\
\hline$r$ & Birth rate (intrinsic growth rate) of the susceptible class \\
\hline$k$ & Carrying capacity of the system \\
\hline$\beta$ & Transmission rate of infection \\
\hline$\alpha$ & Inhibitory coefficient \\
\hline$d$ & Natural death rate of the population \\
\hline$\gamma$ & Rate at which the recovered class loses immunity and becomes susceptible \\
\hline$\gamma$ & Rate at which the infected individuals recovered \\
\hline
\end{tabular}

Lemma 1. The basic reproduction number for the model (1) is

$$
R_{0}=\frac{\beta S_{1}}{\left(1+\alpha S_{1}\right)(d+\gamma)}
$$

Proof. Here is only one infected compartment, that is variable $I$ and DFE is $E_{1}\left(S_{1}, 0,0\right)$. The basic reproduction number $R_{0}$ is defined as the spectral radius of the next generation matrix $F V^{-1}$ with small domain where

$$
\begin{gathered}
F=\left(\frac{\beta S_{1}}{1+\alpha S_{1}}\right)_{1 \times 1} \\
J(S, I, R)=\left(\begin{array}{c}
r-\frac{2 r S}{k}-d-\frac{\beta I}{(1+\alpha S)^{2}} \\
\frac{\beta I}{(1+\alpha S)^{2}} \\
0
\end{array}\right.
\end{gathered}
$$

\section{1. Local Stability of Equilibria}

Now we establish the following theorem to show that $E_{0}, E_{1}$ and $E_{2}$ are locally asymptotically stable equilibrium points under some conditions on parameters.

Theorem 1. The equilibrium point $E_{0}$ is stable if $r \leq d$ and is unstable if $r>d$.

Proof. The eigenvalues of the variational matrix at the point $E_{0}(0,0,0)$ are $-(d-r),-(d+\gamma),-(d+\mu)$. So $E_{0}$ is asymptotically stable for $r<d$ and is unstable for $r>d$. When $r=d$ the eigenvalues of the variational matrix are $0,-(d+\gamma),-(d+\mu)$. So in this case $E_{0}$ is a nonhyperbolic critical point. So we can apply the Centre Manifold Theory to determine its stability. The system (1) can be written as

$$
\frac{d X}{d t}=A X+F(S, I, R)
$$

where

$$
X=\left(\begin{array}{c}
S \\
I \\
R
\end{array}\right)
$$

and

$$
V=(d+\gamma)_{1 \times 1}
$$

[21]. Thus, $R_{0}$ for the model is $\frac{\beta S_{1}}{\left(1+\alpha S_{1}\right)(d+\gamma)}$.

\section{STABILITY OF EQUILIBRIA}

In this section we investigate the local stability and global stability of the equilibrium points. Here the variational matrix corresponding to (1) is

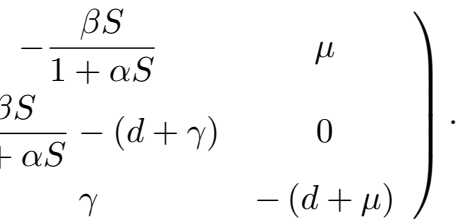

$$
\begin{aligned}
& A=\left(\begin{array}{ccc}
0 & 0 & \mu \\
0 & -(d+\gamma) & 0 \\
0 & \gamma & -(d+\mu)
\end{array}\right) \text {, } \\
& F(S, I, R)=\left(\begin{array}{c}
-\frac{d}{k} S^{2}-\beta S I \\
\beta S I \\
0
\end{array}\right) .
\end{aligned}
$$

(Here we expand $(1+\alpha S)^{-1}$ and we neglect the terms of order greater or equal to 3 ).

Clearly the matrix $A$ is diagonalisable. Thus we can find a matrix

$$
P=\left(\begin{array}{ccc}
1 & -\frac{\mu}{d+\gamma} & 1 \\
0 & \frac{\mu-\gamma}{\gamma} & 0 \\
0 & 1 & -\frac{d+\mu}{\mu}
\end{array}\right)
$$

so that

$$
P^{-1} A P=D=\operatorname{diag}(0,-(d+\gamma),-(d+\mu))
$$


By using the transformation $X=P Y$, where

$$
Y=\left(\begin{array}{c}
S^{\prime} \\
I^{\prime} \\
R^{\prime}
\end{array}\right)
$$

the system (2) can be put in the form (omitting the 'dash' sign)

$$
\begin{gathered}
\frac{d S}{d t}=0+g_{11}(S, I, R) \\
\frac{d I}{d t}=-(d+\gamma) I+g_{22}(S, I, R) \\
\frac{d R}{d t}=-(d+\mu) R+g_{33}(S, I, R), \text { where } \\
g_{11}=-\frac{d}{k}\left(S-\frac{\mu}{d+\gamma} I+R\right)^{2} \\
+\beta\left\{\frac{\mu \gamma}{(d+\mu)(d+\gamma)}-1\right\} \\
\quad \times\left(S-\frac{\mu}{d+\gamma} I+R\right)\left(\frac{\mu-\gamma}{\gamma}\right) I \\
\equiv A_{11} S^{2}+B_{11} I^{2}+C_{11} R^{2} \\
+D_{11} S I+E_{11} S R+F_{11} I R \\
g_{22}=\frac{\gamma \beta}{\mu-\gamma}\left(S-\frac{\mu}{d+\gamma} I+R\right)\left(\frac{\mu-\gamma}{\gamma}\right) I \equiv \\
\equiv B_{22} I^{2}+D_{22} S I+F_{22} I R, \\
g_{33}=\frac{\mu \gamma \beta}{(\mu-\gamma)(d+\mu)}\left(S-\frac{\mu}{d+\gamma} I+R\right)\left(\frac{\mu-\gamma}{\gamma}\right) I \equiv \\
\equiv B_{33} I^{2}+D_{33} S I+F_{33} I R
\end{gathered}
$$

(Where $A_{11}=-d / k$ and other components will be similarly determined).

By the Centre Manifold Theory there exists a centre manifold of the system (3) which can be expressed by $W^{c}(0)=$ $\left\{(S, I, R) / I=h_{1}(S), R=h_{2}(S)\right.$ for $\left.S<\delta\right\}$, where $\delta(>0)$ is some number, $h_{1}(0)=h_{2}(0)=0, D h_{1}(0)=$ $D h_{2}(0)=0$.

To compute the centre manifold $W^{c}(0)$, we assume that $I=h_{1}(S)=h_{11} S^{2}+h_{12} S^{3}+\ldots$ and $R=h_{2}(S)=$ $h_{21} S^{2}+h_{22} S^{3}+\ldots$

So from the Local Centre Manifold Theorem [23], we have the flow on the centre manifold $W^{c}(0)$ defined by the differential equation $\frac{d S}{d t}=A_{11} S^{2}$.

Since $A_{11}=-\frac{d}{k}$ is negative quantity, hence for $r=d$ the system is locally asymptotically stable. Hence the theorem is proved.

Note: Other components $B_{11}, C_{11}, D_{11}, E_{11}, F_{11}, B_{22}, D_{22}$, $F_{22}, B_{33}, D_{33}$ and $F_{33}$ are not derived here as they are not in use.
Theorem 2. If $r>d$ the disease free equilibrium point $E_{1}\left(S_{1}, 0,0\right)$ is locally asymptotically stable for $R_{0}<1$ and is unstable for $R_{0}>1$.

Proof. The equilibrium point $E_{1}$ exists only when $r>d$ and eigenvalues of the Jacobian matrix of the system (1) at $E_{1}$ are $-(r-d),-(d+\mu)$ and $(d+\gamma)\left(R_{0}-1\right)$. Thus $E_{1}$ is asymptotically stable for $R_{0}<1$ and is unstable for $R_{0}>1$. Hence the theorem is proved.

Theorem 3. If $r>d$ and $R_{0}=1$ then $E_{1}\left(S_{1}, 0,0\right)$ is an unstable equilibrium point.

Proof. For $R_{0}=1$, the eigenvalues of the variational matrix of (1) at $E_{1}$ are $0,-(r-d),-(d+\mu)$. Thus $E_{1}$ is a nonhyperbolic critical point and the Centre Manifold Theory will be applied to determine its stability.

We put $S^{\prime}=S-S_{1}, I^{\prime}=I, R^{\prime}=R$ in (1) and we get (omitting the 'dash' sign)

$$
\frac{d X}{d t}=A X+F(S, I, R),
$$

where

$$
\begin{gathered}
X=\left(\begin{array}{c}
S \\
I \\
R
\end{array}\right), \\
A=\left(\begin{array}{ccc}
(d-r) & -(d+\gamma) & \mu \\
0 & 0 & 0 \\
0 & \gamma & -(d+\mu)
\end{array}\right), \\
F(S, I, R)=\left(\begin{array}{c}
-\frac{r}{k} S^{2}-\frac{\beta}{\left(1+\alpha S_{1}\right)^{2}} I S \\
\frac{\beta}{\left(1+\alpha S_{1}\right)^{2}} I S \\
0
\end{array}\right) .
\end{gathered}
$$

Thus we can find a matrix

$$
P=\left(\begin{array}{ccc}
d(d+\gamma+\mu) & 1 & \mu \\
(d+\mu)(d-r) & 0 & 0 \\
\gamma(d-r) & 0 & r-2 d-\mu
\end{array}\right)
$$

so that

$$
P^{-1} A P=\operatorname{diag}(0, d-r,-(d+\mu)) .
$$

By using the transformation $X=P Y$, where

$$
Y=\left(\begin{array}{c}
S^{\prime} \\
I^{\prime} \\
R^{\prime}
\end{array}\right)
$$

system (4) can be transformed into the form (omitting the 'dash' sign)

$$
\begin{aligned}
& \frac{d S}{d t}=0+g_{11}(S, I, R) \\
& \frac{d I}{d t}=-(r-d) I+g_{22}(S, I, R) \\
& \frac{d R}{d t}=-(d+\mu) R+g_{33}(S, I, R), \text { where }
\end{aligned}
$$




$$
g_{11} \equiv A_{11} S^{2}+B_{11} I S+C_{11} R S
$$

$$
g_{22} \equiv A_{22} S^{2}+B_{22} I^{2}+C_{22} R^{2}+D_{22} S I+E_{22} S R+F_{22} I R,
$$$$
g_{33} \equiv A_{33} S^{2}+B_{33} S I+C_{33} S R \text {, where }
$$

$$
A_{11}=\frac{\beta d(d+\gamma+\mu)}{\left(1+\alpha S_{1}\right)^{2}}
$$

and other components will be similarly determined.

Now, we continue the process given in Theorem 1 and we get the flow on the centre manifold $W^{c}(0)$, which is defined by the differential equation

$$
\begin{aligned}
\frac{d S}{d t} & =A_{11} S^{2}+S\left\{B_{11}\left(\frac{A_{22}}{r-d} S^{2}+\ldots\right)\right. \\
& \left.+C_{11}\left(\frac{A_{33}}{d+\mu} S^{2}+\ldots\right)\right\} .
\end{aligned}
$$

Since $A_{11}>0$, hence $E_{1}$ is unstable. Hence the theorem is proved.

Theorem 4. If $1<\Delta_{1}<1+\frac{2}{k \alpha}, \Delta_{2}>1$ and $r>d$, then the endemic equilibrium point $E_{2}\left(S_{2}, I_{2}, R_{2}\right)$ will be locally asymptotically stable.

Proof. $E_{2}$ exists only when $\Delta_{1}>1$ and $\Delta_{2}>1$. The characteristic equation of the variational matrix of (1) at $E_{2}\left(S_{2}, I_{2}, R_{2}\right)$ is

$$
\lambda^{3}+C_{1} \lambda^{2}+C_{2} \lambda+C_{3}=0,
$$

where

$$
\begin{gathered}
C_{1}=2 d+\mu+\frac{\beta I_{2}}{\left(1+\alpha S_{2}\right)^{2}}-\frac{r}{\Delta_{1}-1}\left\{\Delta_{1}-\left(1+\frac{2}{k \alpha}\right)\right\}, \\
C_{2}=\frac{\beta I_{2}(2 d+\gamma+\mu)}{\left(1+\alpha S_{2}\right)^{2}} \\
+(d+\mu)\left[d-\frac{r}{\Delta_{1}-1}\left\{\Delta_{1}-\left(1+\frac{2}{k \alpha}\right)\right\}\right], \\
C_{3}=\frac{\beta I_{2} d(d+\gamma+\mu)}{\left(1+\alpha S_{2}\right)^{2}}
\end{gathered}
$$

and

$$
\begin{aligned}
& C_{1} C_{2}-C_{3}=\frac{\beta^{2} I_{2}^{2}(2 d+\gamma+\mu)}{\left(1+\alpha S_{2}\right)^{4}} \\
+ & \frac{\beta I_{2}}{\left(1+\alpha S_{2}\right)^{2}}\left[-\frac{r}{\Delta_{1}-1}\left\{\Delta_{1}-\left(1+\frac{2}{k \alpha}\right)\right\}\right. \\
\times & \left.(3 d+2 \mu+\gamma)+(2 d+\mu)^{2}+\gamma(d+\mu)\right] \\
+ & (d+\mu)\left[\left(-r+\frac{2 r S_{2}}{k}\right)^{2}-\frac{r}{\Delta_{1}-1}\right. \\
\times & \left.\left\{\Delta_{1}-\left(1+\frac{2}{k \alpha}\right)\right\}(3 d+\mu)+d(2 d+\mu)\right] .
\end{aligned}
$$

Using the conditions stated in the theorem it can be easily shown that the Routh-Hurwitz criterion is satisfied. Hence the theorem is proved.

The stability conditions in Theorem 4 are only sufficient, but not necessary. Thus, if the conditions stated in Theorem 4 are satisfied then the disease will persist in the system. It can be noted that one can easily prove analytically that $E_{0}$ and $E_{1}$ are unstable whenever $E_{2}$ exists. Because $E_{2}$ exists if and only if $\Delta_{1}>1, \Delta_{2}>1$ and these two imply that $r>d$ and basic reproduction number $R_{0}>1$. This is very significant from the biological point of view, because the analysis implies that the coexistence of two stable non-zero steady states is not possible. It means that there is no hysteresis loop. The existence conditions and stability criteria of three equilibrium points have been summarized in the following table.

\section{2. Global Stability of Equilibria}

Next, we shall obtain sufficient conditions on model parameters for the global stability of the disease free equilibrium point $E_{1}\left(S_{1}, 0,0\right)$ and the endemic equilibrium point $E_{2}\left(S_{2}, I_{2}, R_{2}\right)$.

Theorem 5. If $r>d, \Delta_{1}>1$ and $R_{0}<1$, then disease free equilibrium point $E_{1}\left(S_{1}, 0,0\right)$ is globally asymptotically stable in the domain $D_{1}=\left\{(S, I, R) \in R_{+}^{3}: S<\frac{d+\gamma}{\beta-\alpha(d+\gamma)}\right\}$.

Proof. We define a Liapunov function $L=I$. Then $\frac{d L}{d t}=\left\{\frac{\beta S}{1+\alpha S}-(d+\gamma)\right\} I \leq 0$, if $S<\frac{d+\gamma}{\beta-\alpha(d+\gamma)}$. i.e. $\frac{d L}{d t} \leq 0$ in the domain $D_{1}$. So for the positive definite function $L$, the derivative $\frac{d L}{d t}$ is negative semi definite in $D_{1}$. Now we consider the set where $\frac{d L}{d t}=0$.

Let $\Gamma=\left\{(S, I, R) \in D_{1}: \frac{d L}{d t}=0\right\}=\left\{(S, I, R) \in D_{1}\right.$ : $I=0\}$.

Let $M$ be the largest invariant set in $\Gamma$. Then in $\Gamma$, we have

$$
\begin{aligned}
& \frac{d S}{d t}=r S\left(1-\frac{S}{k}\right)-d S+\mu R, \\
& \frac{d R}{d t}=-(d+\mu) R .
\end{aligned}
$$

From the second equation, we have $R \rightarrow 0$ as $t \rightarrow \infty$. Then from the first equation, we have

$$
\frac{d S}{d t}=S\left(r-d-\frac{r}{k} S\right)
$$

when $t \rightarrow \infty$. Since $r>d$, hence $S \rightarrow k\left(1-\frac{d}{r}\right)=S_{1}$ as $t \rightarrow \infty$.

Hence $M$ is singleton $\left\{\left(S_{1}, 0,0\right)\right\}$. Then, it follows from the LaSalle-Liapunov theory, [22], that $E_{1}\left(S_{1}, 0,0\right)$ is globally asymptotically stable in $D_{1}$. Hence the theorem is proved.

Thus, we are able to establish the conditions for which the disease free equilibrium point is globally asymptotically stable. In the next theorem we devote our attention to discuss 
Tab. 2. Feasibility and local stability conditions of equilibrium points

\begin{tabular}{c|c|c}
\hline Equilibrium points & Existence conditions & Stability conditions \\
\hline$E_{0}(0,0,0)$ & Always exists & Stable if $r \leq d$,unstable if $r>d$ \\
\hline$E_{1}\left(S_{1}, 0,0\right)$ & Exists if and only if $r>d$ & Stable if $R_{0}<1$, unstable if $R_{0} \geq 1$ \\
\hline$E_{2}\left(S_{2}, I_{2}, R_{2}\right)$ & Exists if and only if $\Delta_{1}>1, \Delta_{2}>1$ & Stable if $\Delta_{1}<1+\frac{2}{k \alpha}$ \\
\hline
\end{tabular}

the global stability of the endemic equilibrium point in the region $D_{2}$.

Theorem 6. If

$$
I_{2}<\frac{r}{k \alpha \beta},
$$

then the system is said to be globally asymptotically stable around the equilibrium point $E_{2}\left(S_{2}, I_{2}, R_{2}\right)$ in the region $D_{2}=\left\{(S, I, R) \in R_{+}^{3}: 1<\frac{I_{2}}{I}<\frac{R_{2}}{R}<\frac{S_{2}}{S}\right\}$ when $E_{2}$ exists and also the system has no closed orbit in $D_{2}$.

Proof. We consider a Liapunov function $L$ defined as follows

$$
L=\int_{S_{2}}^{S} \frac{S-S_{2}}{S} d S+\int_{I_{2}}^{I} \frac{I-I_{2}}{I} d I+\int_{R_{2}}^{R} \frac{R-R_{2}}{R} d R .
$$

Then

$$
\begin{aligned}
& \frac{d L}{d t}=\left(\frac{S-S_{2}}{S}\right) \frac{d S}{d t}+\left(\frac{I-I_{2}}{I}\right) \frac{d I}{d t}+\left(\frac{R-R_{2}}{R}\right) \frac{d R}{d t} \\
= & \left(S-S_{2}\right)\left\{r\left(1-\frac{S}{k}\right)-\frac{\beta I}{1+\alpha S}+\frac{\mu R}{S}-r\left(1-\frac{S_{2}}{k}\right)\right. \\
+ & \left.\frac{\beta I_{2}}{1+\alpha S_{2}}-\frac{\mu R_{2}}{S_{2}}\right\}+\left(I-I_{2}\right)\left\{\frac{\beta S}{1+\alpha S}-\frac{\beta S_{2}}{1+\alpha S_{2}}\right\} \\
+ & \left(R-R_{2}\right)\left\{\frac{\gamma I}{R}-\frac{\gamma I_{2}}{R_{2}}\right\}=-\frac{r}{k}\left(S_{2}-S\right)^{2} \\
+ & \frac{\alpha \beta\left(S_{2}-S\right)\left(I S_{2}-S I_{2}\right)}{\left(1+\alpha S_{2}\right)(1+\alpha S)}-\frac{\gamma\left(R_{2}-R\right)\left(I R_{2}-I_{2} R\right)}{R R_{2}} \\
- & \frac{\mu\left(S_{2}-S\right)\left(R S_{2}-R_{2} S\right)}{S S_{2}} .
\end{aligned}
$$

Since $1<\frac{I_{2}}{I}<\frac{R_{2}}{R}<\frac{S_{2}}{S}$, hence $\frac{S_{2}}{S}-\frac{I_{2}}{I}<\frac{S_{2}}{S}-1$ i.e. $\left(I S_{2}-S I_{2}\right)<I\left(S_{2}-S\right)$.

So

$$
\begin{aligned}
& \frac{d L}{d t}<-\frac{r}{k}\left(S_{2}-S\right)^{2}+\frac{\alpha \beta\left(S_{2}-S\right)^{2} I}{\left(1+\alpha S_{2}\right)(1+\alpha S)} \\
- & \frac{\gamma\left(R_{2}-R\right)\left(I R_{2}-I_{2} R\right)}{R R_{2}}-\frac{\mu\left(S_{2}-S\right)\left(R S_{2}-R_{2} S\right)}{S S_{2}}< \\
- & \frac{r}{k}\left(S_{2}-S\right)^{2}+\alpha \beta\left(S_{2}-S\right)^{2} I-\frac{\gamma\left(R_{2}-R\right)\left(I R_{2}-I_{2} R\right)}{R R_{2}} \\
- & \frac{\mu\left(S_{2}-S\right)\left(R S_{2}-R_{2} S\right)}{S S_{2}}<-\left(S_{2}-S\right)^{2}\left\{\frac{r}{k}-\alpha \beta I_{2}\right\} \\
- & \frac{\gamma\left(R_{2}-R\right)\left(I R_{2}-I_{2} R\right)}{R R_{2}}-\frac{\mu\left(S_{2}-S\right)\left(R S_{2}-R_{2} S\right)}{S S_{2}}<0
\end{aligned}
$$

in the region $D_{2}$, if $I_{2}<\frac{r}{k \alpha \beta}$. Hence the theorem is proved.
Thus, there exists a region where the endemic equilibrium point is globally asymptotically stable. In Theorem 5 and Theorem 6 we obtain the conditions for which the equilibrium points are globally asymptotically stable. Biologically, globally asymptotically stable means whatever the initial number of $S, I, R$, the system finally will converge to the equilibrium point. From these two theorems one can conclude that despite the initial number of infected population, the system will converge to the corresponding equilibrium point when the conditions are satisfied.

Now we shall discuss the particular case in absence of the inhibitory factor. Then the reduced equilibrium point $E_{2}\left(S_{2}, I_{2}, R_{2}\right)$ is globally asymptotically stable in some region.

Theorem 7. For $\alpha=0$ the system is said to be globally asymptotically stable around the equilibrium point $E_{2}\left(S_{2}, I_{2}, R_{2}\right)$ in the region

$$
D_{3}=\left\{(S, I, R) \in R_{+}^{3}: 1<\frac{I_{2}}{I}<\frac{R_{2}}{R}<\frac{S_{2}}{S}\right\}
$$

if $E_{2}$ exists.

Proof. We choose a Liapunov function $L$ defined as follows

$$
L=\int_{S_{2}}^{S} \frac{S-S_{2}}{S} d S+\int_{I_{2}}^{I} \frac{I-I_{2}}{I} d I+\int_{R_{2}}^{R} \frac{R-R_{2}}{R} d R .
$$

Then

$$
\begin{aligned}
\frac{d L}{d t} & =\left(\frac{S-S_{2}}{S}\right) \frac{d S}{d t}+\left(\frac{I-I_{2}}{I}\right) \frac{d I}{d t}+\left(\frac{R-R_{2}}{R}\right) \frac{d R}{d t} \\
& =\left(S-S_{2}\right)\left\{r\left(1-\frac{S}{k}\right)-\beta I-d+\frac{\mu R}{S}\right\} \\
& +\left(I-I_{2}\right)\{\beta S-(d+\gamma)\} \\
& +\left(R-R_{2}\right)\left\{\frac{\gamma I}{R}-(d+\mu)\right\} \\
& =-\frac{r}{k}\left(S_{2}-S\right)^{2}-\frac{\gamma\left(R_{2}-R\right)\left(I R_{2}-I_{2} R\right)}{R R_{2}} \\
& -\frac{\mu\left(S_{2}-S\right)\left(R S_{2}-R_{2} S\right)}{S S_{2}}<0
\end{aligned}
$$

in the region $D_{3}$. Hence the theorem is proved.

When the recovered class does not losse immunity and so does not become susceptible, then the reduced equilibrium point $E_{2}\left(S_{2}, I_{2}, R_{2}\right)$ will be globally asymptotically stable. Lemma 2. If $k \alpha<1$, the system does not have a closed orbit in the first quadrant of $(S, I)$ plane in the case $\mu=0$. 
Proof. If $\mu=0$, then the system (1) is reduced to

$$
\begin{aligned}
& \frac{d S}{d t}=r S\left(1-\frac{S}{k}\right)-\frac{\beta S I}{1+\alpha S}-d S \equiv F(S, I), \\
& \frac{d I}{d t}=\frac{\beta S I}{1+\alpha S}-(d+\gamma) I \equiv G(S, I) .
\end{aligned}
$$

Now, taking the Dulac function $B(S, I)=\frac{1+\alpha S}{S I}$, we obtain

$$
\begin{aligned}
& \frac{\partial}{\partial S}(B F)+\frac{\partial}{\partial I}(B G)= \\
= & \frac{r\left\{\left(1-\frac{S}{k}\right) \alpha-(1+\alpha S) \frac{1}{k}\right\}-d \alpha}{I}<0 \text { if } k \alpha<1 .
\end{aligned}
$$

This proves the lemma.

Theorem 8. If $1<\Delta_{1}<1+\frac{2}{k \alpha}, \Delta_{2}>1$ and $k \alpha<1$, then the endemic equilibrium point $E_{2}\left(S_{2}, I_{2}, R_{2}\right)$ will be globally asymptotically stable in the case $\mu=0$.

Proof. In Theorem 4, it has been proved that $E_{2}$ is locally asymptotically stable if $1<\Delta_{1}<1+\frac{2}{k \alpha}$ and $\Delta_{2}>1$. Thus, it follows from Lemma 2 that $E_{2}$ is globally asymptotically stable.

\section{BIFURCATION ANALYSIS OF THE MODEL}

A bifurcation is a qualitative change in the behaviour of solutions as one or more parameters are varied. A bifurcation is called local bifurcation if the qualitative change occurs in the neighbourhood of an equilibrium point or periodic solution. Here we present two local bifurcations: one is Transcritical bifurcation and the other is Hopf bifurcation.

Theorem 9. If $\beta k(r-d)>r(d+\gamma)$, then the system (1) experiences Transcritical bifurcation at the disease free equilibrium point $E_{1}\left(S_{1}, 0,0\right)$ with respect to the inhibitory factor $\alpha$.

Proof. Let

$$
\begin{gathered}
f(S, I, R ; \alpha)=\left(\begin{array}{c}
r S\left(1-\frac{S}{k}\right)-\frac{\beta S I}{1+\alpha S}-d S+\mu R \\
\frac{\beta S I}{1+\alpha S}-(d+\gamma) I \\
\gamma I-(d+\mu) R
\end{array}\right), \\
\alpha_{0}=\frac{\beta}{d+\gamma}-\frac{r}{k(r-d)}
\end{gathered}
$$

and

$A=D f\left(E_{1}, \alpha_{0}\right)=\left(\begin{array}{ccc}(d-r) & -\frac{\beta S_{1}}{1+\alpha S_{1}} & \mu \\ 0 & 0 & 0 \\ 0 & \gamma & -(d+\mu)\end{array}\right)$.

Clearly, $f\left(E_{1}, \alpha_{0}\right)=0$ and $A$ has a simple eigenvalue $\lambda=0$. So we shall use Sotomayor theorem [23] to check the nature of solutions for $\alpha=\alpha_{0}$.
Now, the eigenvectors of $A$ and $A^{T}$ corresponding to the eigenvalue $\lambda=0$ are, respectively,

$$
V=\left(\begin{array}{c}
\beta S_{1}(d+\mu)-\mu \gamma\left(1+\alpha S_{1}\right) \\
(d+\mu)\left(1+\alpha S_{1}\right)(d-r) \\
\gamma\left(1+\alpha S_{1}\right)(d-r)
\end{array}\right)
$$

and

$$
W=\left(\begin{array}{l}
0 \\
1 \\
0
\end{array}\right) .
$$

Let $f_{\alpha}$ denote the vector of partial derivatives of the components of $f$ with respect to $\alpha$.

Thus

$$
f_{\alpha}=\left(\begin{array}{c}
\frac{\beta S^{2} I}{(1+\alpha S)^{2}} \\
-\frac{\beta S^{2} I}{(1+\alpha S)^{2}} \\
0
\end{array}\right)
$$

and so

$$
f_{\alpha}\left(E_{1}, \alpha_{0}\right)=\left(\begin{array}{l}
0 \\
0 \\
0
\end{array}\right) .
$$

Therefore, $W^{T} f_{\alpha}\left(E_{1}, \alpha_{0}\right)=(0)$.

Again,

$$
\begin{aligned}
& W^{T}\left[D f_{\alpha}\left(E_{1}, \alpha_{0}\right) V\right]= \\
= & (0,1,0)\left(\begin{array}{c}
-\frac{(d+\gamma)(d+\mu)(r-d)^{2} k}{r} \\
\frac{(d+\gamma)(d+\mu)(r-d)^{2} k}{r} \\
0
\end{array}\right)= \\
= & \left(\frac{(d+\gamma)(d+\mu)(r-d)^{2} k}{r}\right) \neq(0) .
\end{aligned}
$$

Finally,

$$
\begin{aligned}
& W^{T}\left[D^{2} f\left(E_{1}, \alpha_{0}\right)(V, V)\right]= \\
= & \left(\frac{2 \beta(d+\mu)(d-r)\left\{\beta S_{1}(d+\mu)-\mu \gamma\left(1+\alpha_{0} S_{1}\right)\right\}}{\left(1+\alpha_{0} S_{1}\right)}\right) \\
\neq & (0) .
\end{aligned}
$$

Therefore, all the conditions for Transcritical bifurcation in Sotomayor theorem are satisfied. Hence, the system (1) experiences a Transcritical bifurcation at the equilibrium point $E_{1}\left(S_{1}, 0,0\right)$ as the parameter $\alpha$ varies through the bifurcation value $\alpha=\alpha_{0}$. Hence the theorem is proved.

Now we consider the other possible scenario, where several limit cycles bifurcate from an equilibrium point. We shall vary $r$ in the system to obtain a Hopf bifurcation around the endemic equilibrium point $E_{2}$. In Theorem 4, we see that the characteristic equation of the Jacobian matrix of the system (1) at the equilibrium point $E_{2}$ is $\lambda^{3}+C_{1} \lambda^{2}+C_{2} \lambda+C_{3}=0$. Since the expressions of $C_{1}, C_{2}, C_{3}$ and $C_{1} C_{2}-C_{3}$ depend 
on intrinsic growth rate $r$, hence the sign of $C_{1}, C_{2}, C_{3}$ and $C_{1} C_{2}-C_{3}$ can be controlled by changing the values of $r$. For Hopf bifurcation around $E_{2}$ at $r=r_{c}$, we must have $C_{1}\left(r_{c}\right) C_{2}\left(r_{c}\right)-C_{3}\left(r_{c}\right)=0$ and $C_{1}, C_{2}, C_{3}$ are all positive. For $r \in\left(r_{c}-\varepsilon, r_{c}+\varepsilon\right)$, the roots are, in general, of the form

$$
\begin{aligned}
& y_{1}(r)=\alpha_{0}(r)+i \beta_{0}(r), \\
& y_{2}(r)=\alpha_{0}(r)-i \beta_{0}(r), \\
& y_{3}(r)=-C_{1}(r) .
\end{aligned}
$$

To apply the Hopf bifurcation theorem, we need to verify the transversality condition

$$
\operatorname{Re}\left[\frac{d y_{i}}{d r}\right]_{r=r_{c}} \neq 0, i=1,2
$$

which is equivalent to $\left[\left(C_{1} C_{2}^{\prime}+C_{1}^{\prime} C_{2}-C_{3}^{\prime}\right)\right]_{r=r_{c}} \neq 0$.

We choose the critical value of $r$, say $r_{c}$, in such a manner that $C_{1} C_{2}-C_{3}=0$ and $\left[\left(C_{1} C_{2}^{\prime}+C_{1}^{\prime} C_{2}-C_{3}^{\prime}\right)\right] \neq 0$ at $r=r_{c}$ which will hold if $\Delta_{1}>1+\frac{2}{k \alpha}$ and $\Delta_{2}>1$. Thus we summarize the details in the following:

Theorem 10. If $\Delta_{1}>1+\frac{2}{k \alpha}$ and $\Delta_{2}>1$, then the system may exhibit a Hopf bifurcation leading to a family of periodic solutions that bifurcates from $E_{2}$ for suitable values of intrinsic growth rate $r$ of the susceptible class in a neighbourhood of $r_{c}$.

Next, we check the stability of the bifurcating periodic orbits. We know that the periodic solutions are stable for supercritical bifurcation, and they are unstable for subcritical bifurcation. We need to compute the index number $\Gamma$ in the Hopf bifurcation theorem by using the Centre Manifold Theorem. We first translate the equilibrium point $E_{2}\left(S_{2}, I_{2}, R_{2}\right)$ to the origin. So we put $S^{\prime}=S-S_{2}, I^{\prime}=I-I_{2}$ and $R^{\prime}=R-R_{2}$ in (1) and we get (omitting the 'dash' sign)

$$
\frac{d X}{d t}=B X+F(X)
$$

Here $X=\left(\begin{array}{c}S \\ I \\ R\end{array}\right), B=\left(\begin{array}{ccc}a_{11} & a_{12} & a_{13} \\ a_{21} & a_{22} & 0 \\ 0 & a_{31} & a_{32}\end{array}\right)$,

$$
F(X)=\left(\begin{array}{c}
a_{14} S^{2}+a_{15} S I \\
a_{23} S^{2}+a_{24} S I+a_{25} I^{2} \\
0
\end{array}\right)
$$

where

$$
\begin{aligned}
& a_{11}=r\left(1-\frac{2 S_{2}}{k}\right)-d-\frac{\beta I_{2}}{\left(1+\alpha S_{2}\right)^{2}}, \\
& a_{12}=-\frac{\beta S_{2}}{1+\alpha S_{2}}, \\
& a_{13}=\mu, \\
& a_{14}=-\frac{r}{k}+\frac{\alpha \beta I_{2}}{\left(1+\alpha S_{2}\right)^{3}}, \\
& a_{15}=-\frac{2 \beta}{\left(1+\alpha S_{2}\right)^{2}}, \\
& a_{21}=\frac{\beta I_{2}}{\left(1+\alpha S_{2}\right)^{2}}, \\
& a_{22}=\frac{\beta S_{2}}{1+\alpha S_{2}}, \\
& a_{23}=-\frac{\alpha \beta I_{2}}{\left(1+\alpha S_{2}\right)^{3}}, \\
& a_{24}=\frac{\beta}{\left(1+\alpha S_{2}\right)^{2}}, \\
& a_{25}=-\frac{(d+\gamma)}{2}, \\
& a_{31}=\gamma, \\
& a_{32}=-(d+\mu) .
\end{aligned}
$$

We know that the eigenvalues of the matrix $B$ are $\lambda=$ $\pm \sqrt{C_{2}} i,-C_{1}$ at $r=r_{c}$. So an eigenvector of $B$ corresponding to the eigenvalue

$$
\lambda=\sqrt{C_{2}} i
$$

is

$$
\left(\begin{array}{l}
\alpha_{1} \\
\alpha_{2} \\
\alpha_{3}
\end{array}\right)+i\left(\begin{array}{l}
\beta_{1} \\
\beta_{2} \\
\beta_{3}
\end{array}\right)
$$

and corresponding to the eigenvalue $\lambda=-C_{1}$ is $\left(\begin{array}{l}\gamma_{1} \\ \gamma_{2} \\ \gamma_{3}\end{array}\right)$, where

$$
\begin{aligned}
& \alpha_{1}=-\frac{a_{22}}{a_{21}}, \quad \alpha_{2}=1, \quad \alpha_{3}=-\frac{a_{31} \cdot a_{32}}{c_{2}+a_{32}^{2}}, \\
& \beta_{1}=\frac{\sqrt{c_{2}}}{a_{21}}, \quad \beta_{2}=0, \quad \beta_{3}=-\frac{a_{31} \cdot \sqrt{c_{2}}}{c_{2}+a_{32}^{2}}, \\
& \gamma_{1}=-\frac{c_{1}+a_{22}}{a_{21}}, \quad \gamma_{2}=1, \quad \gamma_{3}=-\frac{a_{31}}{c_{1}+a_{32}} .
\end{aligned}
$$

Now, by the transformation $X=P Y$, the system (7) can be written as

$$
\frac{d Y}{d t}=\left(P^{-1} B P\right) Y+P^{-1} F(P Y),
$$




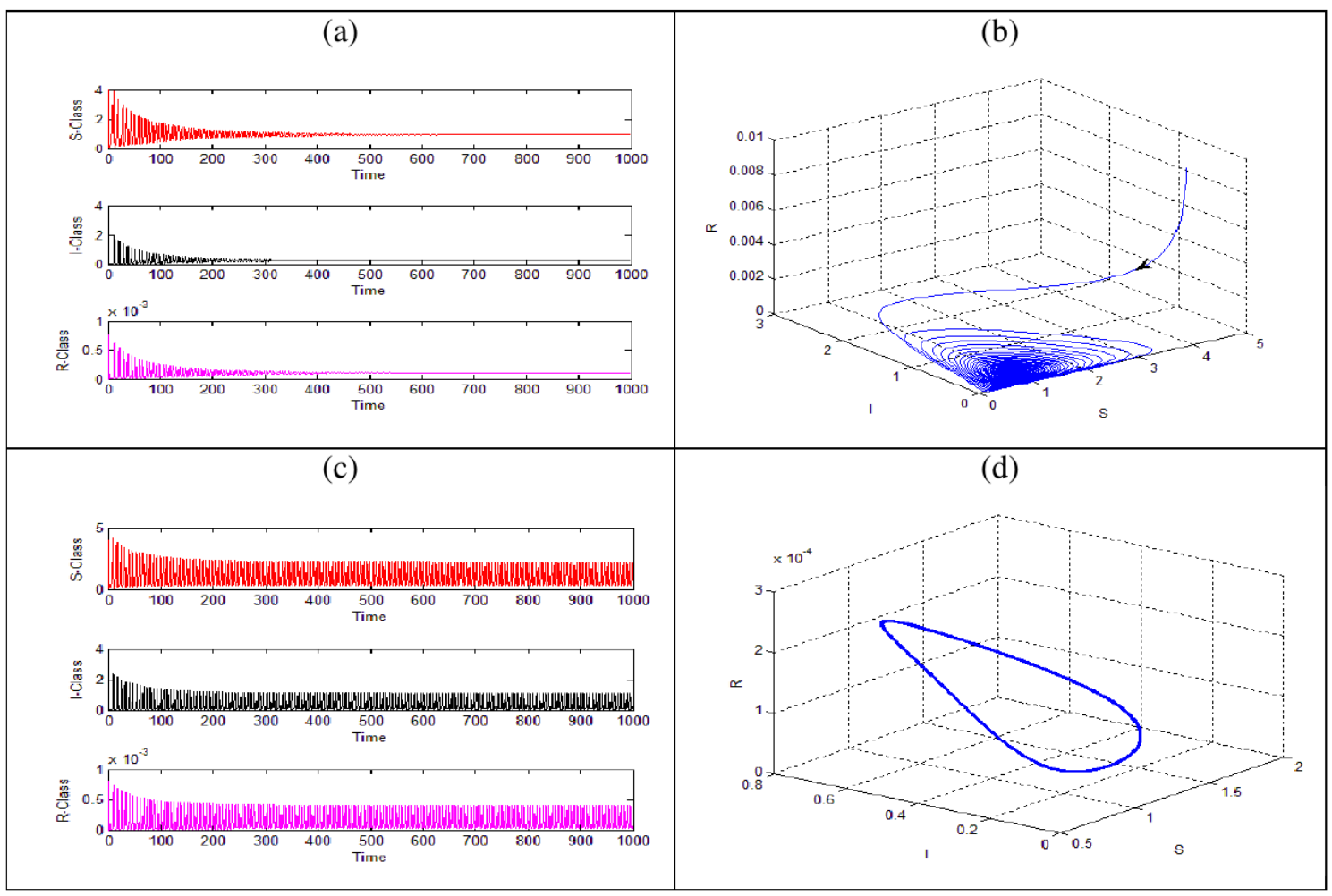

Fig. 1. (a) Time series solutions and (b) phase portrait for the parametric values reported in Table 3 with $r=3.07$, (c) time series solutions and (d) limit cycle for the parametric values reported in Table 3 with $r=3.136$

where

$$
\begin{gathered}
X=\left(\begin{array}{l}
S \\
I \\
R
\end{array}\right), \\
Y=\left(\begin{array}{l}
y_{1} \\
y_{2} \\
y_{3}
\end{array}\right), \\
P=\left(\begin{array}{lll}
\beta_{1} & \alpha_{1} & \gamma_{1} \\
\beta_{2} & \alpha_{2} & \gamma_{2} \\
\beta_{3} & \alpha_{3} & \gamma_{3}
\end{array}\right)
\end{gathered}
$$

and so

$$
P^{-1}=\left(\begin{array}{lll}
p_{11} & p_{12} & p_{13} \\
p_{21} & p_{22} & p_{23} \\
p_{31} & p_{32} & p_{33}
\end{array}\right)
$$

(where $p_{i j}$ can be evaluated from the relation $P P^{-1}=$ $\left.P^{-1} P=I_{3}\right)$.
We rewrite the system (8) as

$$
\begin{aligned}
\left(\begin{array}{l}
\dot{y}_{1} \\
\dot{y}_{2} \\
\dot{y}_{3}
\end{array}\right) & =\left(\begin{array}{ccc}
0 & -\sqrt{C_{2}} & 0 \\
\sqrt{C_{2}} & 0 & 0 \\
0 & 0 & -C_{1}
\end{array}\right) \\
& \times\left(\begin{array}{l}
y_{1} \\
y_{2} \\
y_{3}
\end{array}\right)+\left(\begin{array}{l}
f_{1} \\
f_{2} \\
f_{3}
\end{array}\right) .
\end{aligned}
$$

Here,

$$
\begin{gathered}
f_{1}\left(y_{1}, y_{2}, y_{3}\right)=p_{11}\left(a_{14} S^{2}+a_{15} S I\right) \\
+p_{12}\left(a_{23} S^{2}+a_{24} S I+a_{25} I^{2}\right), \\
f_{2}\left(y_{1}, y_{2}, y_{3}\right)=p_{21}\left(a_{14} S^{2}\right. \\
\left.+a_{15} S I\right)+p_{22}\left(a_{23} S^{2}+a_{24} S I+a_{25} I^{2}\right),
\end{gathered}
$$

$$
\begin{gathered}
f_{3}\left(y_{1}, y_{2}, y_{3}\right)=p_{31}\left(a_{14} S^{2}\right. \\
\left.+a_{15} S I\right)+p_{32}\left(a_{23} S^{2}+a_{24} S I+a_{25} I^{2}\right)
\end{gathered}
$$

where

$$
S=\beta_{1} y_{1}+\alpha_{1} y_{2}+\gamma_{1} y_{3}
$$

$$
I=\beta_{2} y_{1}+\alpha_{2} y_{2}+\gamma_{2} y_{3}
$$




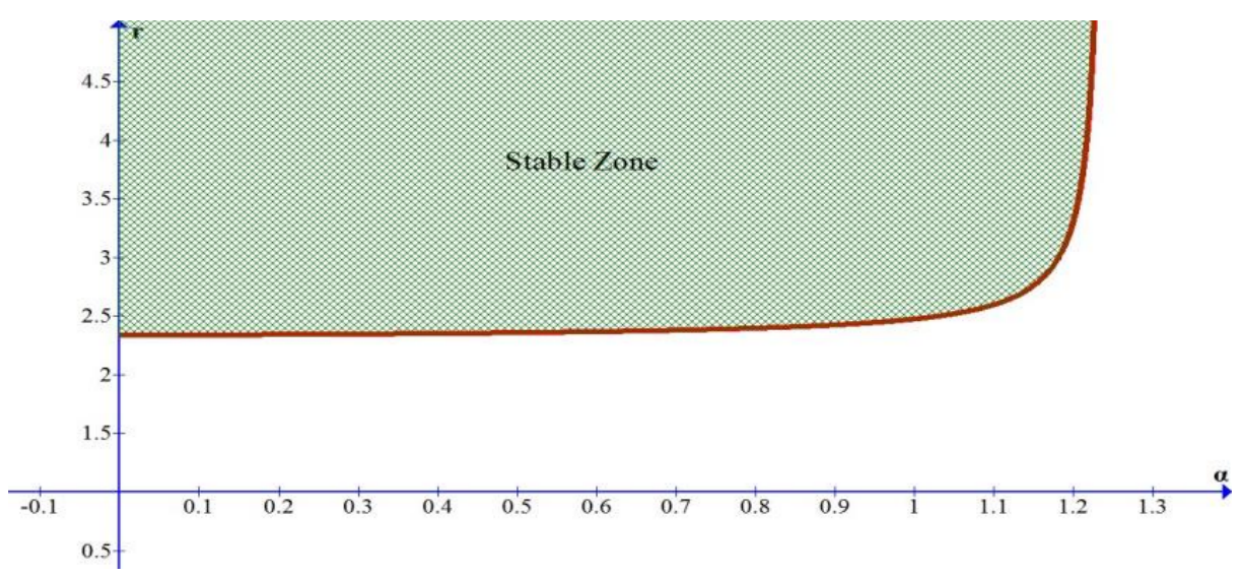

Fig. 2. Stability region for the equilibrium point $E_{1}\left(S_{1}, 0,0\right)$ in $\alpha-r$ plane and value of other parameters $\beta, d, k, \gamma$ reported in Table 4. The red line in the boundary denotes the boundary curve

$$
R=\beta_{3} y_{1}+\alpha_{3} y_{2}+\gamma_{3} y_{3}
$$

By the Centre Manifold Theory there exists a centre manifold of (9), which can be expressed by

$$
W^{c}(0)=\left\{\left(y_{1}, y_{2}, y_{3}\right) / y_{3}=h\left(y_{1}, y_{2}\right)\right.
$$

for

$$
\left.\left|y_{1}\right|<\delta,\left|y_{2}\right|<\delta\right\}
$$

where $\delta(>0)$ is some number. Thus

$$
y_{3}=h\left(y_{1}, y_{2}\right)=k_{1} y_{1}^{2}+k_{2} y_{1} y_{2}+k_{3} y_{2}^{2}+\cdots,
$$

where the constants $k_{i}(i=1,2,3, \ldots)$ can be easily deter- mined from the identity relation

$$
\begin{aligned}
& \left(2 k_{1} y_{1}+k_{2} y_{2}+\cdots\right)\left\{-\sqrt{c_{2}} y_{2}\right. \\
+ & f_{1}\left(y_{1}, y_{2}, k_{1} y_{1}^{2}+k_{2} y_{1} y_{2}+k_{3} y_{2}^{2}\right. \\
+ & \cdots)\}+\left(k_{2} y_{1}+2 k_{3} y_{2}+\cdots\right)\left\{\sqrt{c_{2}} y_{1}\right. \\
+ & \left.f_{2}\left(y_{1}, y_{2}, k_{1} y_{1}^{2}+k_{2} y_{1} y_{2}+k_{3} y_{2}^{2}+\cdots\right)\right\} \\
+ & c_{1}\left(k_{1} y_{1}^{2}+k_{2} y_{1} y_{2}+k_{3} y_{2}^{2}+\cdots\right) \\
- & f_{3}\left(y_{1}, y_{2}, k_{1} y_{1}^{2}+k_{2} y_{1} y_{2}+k_{3} y_{2}^{2}+\cdots\right)=0 .
\end{aligned}
$$

Thus, the flow on the centre manifold has the form

$$
\begin{aligned}
\left(\begin{array}{l}
\dot{y}_{1} \\
\dot{y}_{2}
\end{array}\right) & =\left(\begin{array}{cc}
0 & -\sqrt{C_{2}} \\
\sqrt{C_{2}} & 0
\end{array}\right)\left(\begin{array}{l}
y_{1} \\
y_{2}
\end{array}\right) \\
& +\left(\begin{array}{l}
g^{1}\left(y_{1}, y_{2}\right) \\
g^{2}\left(y_{1}, y_{2}\right)
\end{array}\right),
\end{aligned}
$$

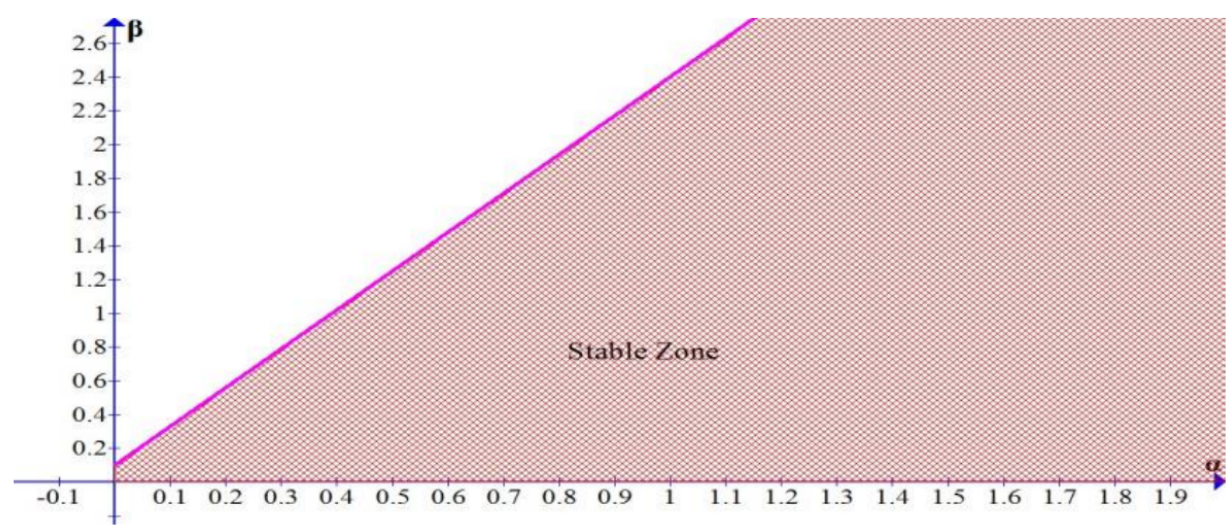

Fig. 3. Stability region for the equilibrium point $E_{1}\left(S_{1}, 0,0\right)$ in $\alpha-\beta$ plane and value of other parameters $r, d, k, \gamma, \mu$ reported in Table 4 . The pink line in the boundary denotes the boundary curve 


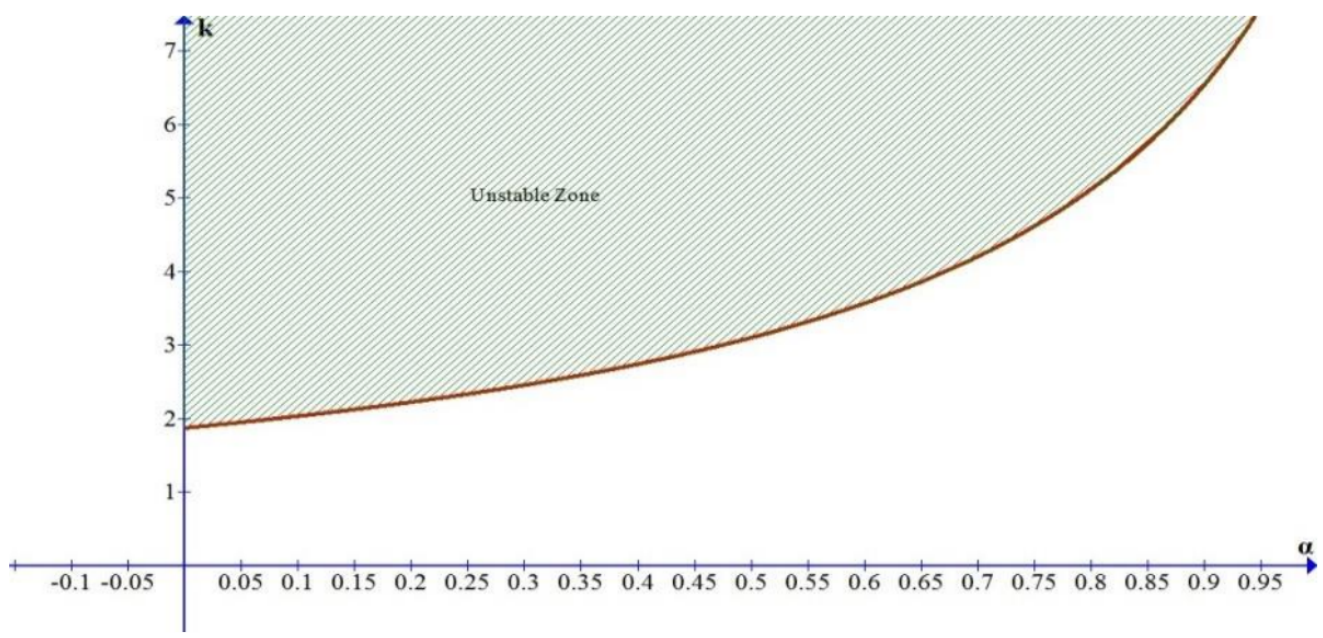

Fig. 4. Instability zone for the equilibrium point $E_{1}\left(S_{1}, 0,0\right)$ in $\alpha-k$ plane and value of other parameters $r, \beta, d, \gamma$ reported in Table 4 . The red line in the boundary denotes the boundary curve

where

$$
\begin{aligned}
& g^{1}\left(y_{1}, y_{2}\right)=f_{1}\left(y_{1}, y_{2}, h\left(y_{1}, y_{2}\right)\right) \\
& g^{2}\left(y_{1}, y_{2}\right)=f_{2}\left(y_{1}, y_{2}, h\left(y_{1}, y_{2}\right)\right)
\end{aligned}
$$

Therefore, we can easily compute the index $\Gamma$, where

$$
\begin{aligned}
\Gamma & =\frac{1}{16}\left\{g_{y_{1} y_{1} y_{1}}^{1}+g_{y_{1} y_{2} y_{2}}^{1}+g_{y_{1} y_{1} y_{2}}^{2}+g_{y_{2} y_{2} y_{2}}^{2}\right\} \\
& +\frac{1}{16 \sqrt{C_{2}}}\left\{g_{y_{1} y_{2}}^{1}\left(g_{y_{1} y_{1}}^{1}+g_{y_{2} y_{2}}^{1}\right)\right. \\
& \left.-g_{y_{1} y_{2}}^{2}\left(g_{y_{1} y_{1}}^{2}+g_{y_{2} y_{2}}^{2}\right)-g_{y_{1} y_{1}}^{1} g_{y_{1} y_{1}}^{2}+g_{y_{2} y_{2}}^{1} g_{y_{2} y_{2}}^{2}\right\} .
\end{aligned}
$$

By using the Hopf bifurcation theorem, we obtain the following result about the periodic solutions bifurcated at $E_{2}$.

Theorem 11. If $\Gamma<0$, the periodic solution in the neighbourhood of the endemic equilibrium point $E_{2}\left(S_{2}, I_{2}, R_{2}\right)$ is stable, while $\Gamma>0$ the periodic solution is unstable.

When $\Gamma<0$, the system is said to be supercritical and the case $\Gamma>0$ is refereed as the subcritical, where $\Gamma$ is defined in the text.

\section{NUMERICAL SIMULATIONS}

In this section, we present some numerical simulation results to exemplify the analytical studies. The values of the parameters are chosen arbitrarily.

Tab. 3. Values of the parameters

\begin{tabular}{c|c|c|c|c|c}
\hline$k$ & $\beta$ & $d$ & $\gamma$ & $\mu$ & $\alpha$ \\
\hline 25 & 2.9 & 2.3 & 0.001 & 0.01 & 0.21 \\
\hline
\end{tabular}

Figure 1(a)-(d) show the existence of Hopf bifurcation about the endemic equilibrium point $E_{2}$ with respect to the intrinsic growth rate $r$ for the given set of parametric values. Figure 1(a)-(b) represent the stable solution for $r<r_{c}$ and in Figure 1(c)-(d), we see that periodic solutions arise for $r=r_{c}(\approx 3.136)$. Hence the system loses stability when $r$ passes through $r=r_{c}$ which suggests that Hopf bifurcation takes place at $r=r_{c}$.

Tab. 4. Values of the parameters

\begin{tabular}{c|c|c|c|c|c}
\hline$k$ & $\beta$ & $d$ & $\gamma$ & $\mu$ & $r$ \\
\hline 55 & 2.9 & 2.3 & 0.001 & 0.01 & 4 \\
\hline
\end{tabular}

If $r>d$, then $E_{1}\left(S_{1}, 0,0\right)$ is stable for $R_{0}<1$ and is unstable for $R_{0} \geq 1$. So $R_{0}=1$ is the boundary curve for the Transcritical bifurcation at the point $E_{1}\left(S_{1}, 0,0\right)$. Figure 2 and 3 represent the stability regions for $E_{1}$ in two parametric domain $(\alpha, r)$ and $(\alpha, \beta)$, respectively. The unstable region for $E_{1}\left(S_{1}, 0,0\right)$ is marked in two parametric domain $(\alpha, k)$ by Figure 4 . We also have indicated the boundary curve in all three figures. The boundary lines in the Figures (2-4) represent the Transcritical bifurcation curves. When we analyse Figure 2, we can see that if we fix $\alpha$ in the range $(0,1.2)$ then the solution in the neighbourhood of disease free equilibrium point $E_{1}\left(S_{1}, 0,0\right)$ is unstable for a low value of the intrinsic growth rate $(r<2.4$ approx) but for $r>2.4$ it is stable and if $\alpha>1.2$ the solution is unstable for all $r$. Again, from Figure 3 we have seen that for the stability of the DFE, the value of inhibitory coefficient has to be high for high rate of infection, and Figure 4 shows that for very low carrying capacity of the system ( $k<1.9$ approx), the DFE is stable for $\alpha=0$ but for $k>1.9$, the inhibitory coefficient has to be increased for the stability of DFE.

Again, $E_{2}\left(S_{2}, I_{2}, R_{2}\right)$ is asymptotically stable if $C_{1}>0$, 
$C_{3}>0$ and $C_{1} C_{2}-C_{3}>0$. For different values of the parameters, the two parametric stability regions are shown in Figure 5(a)-(c) for the endemic equilibrium point $E_{2}\left(S_{2}, I_{2}, R_{2}\right)$ in two parametric domain $(k, r),(\alpha, r)$ and $(\beta, r)$, respectively. Figure 5(a) shows that the endemic equilibrium point $E_{2}$ is stable if $2.39<r<3.136$ for the parametric values given in Table 3 . For these parametric values, $E_{2}$ does not exist if $r<2.39$ and is unstable if $r>3.136$. Again, if we fix the parameters $k, \beta, d, \gamma$ and $\mu$ as listed in Table 3 then Figure 5(b) shows that for every values of $r, E_{2}$ cannot be stable if $\alpha>1.26$. In fact, $E_{2}$ is not feasible for $\alpha>1.26$. Moreover, for $\alpha<1.26$ the region below the blue coloured region represents the infeasible region for $E_{2}$ and the region above the blue coloured region represents the unstable region for the endemic equilibrium point $E_{2}$. Finally, Figure 5(c) indicates $E_{2}$ cannot be stable if $\beta<0.5$ for the other parametric values given in Table 3 . In fact, $E_{2}$ is infeasible for $\beta<0.5$. For $\beta>0.5$ the regions below and above the stable region represent the infeasible and unstable region for $E_{2}$, respectively.

Now, we will present the one-parameter bifurcation diagrams to represent the behaviour of the solutions for different values of the bifurcation parameters $r, \alpha, \beta, k$.

To illustrate the effect of intrinsic growth rate $r$, we have plotted all the populations $S, I$ and $R$ (cf. Figure 6) as the variation of the values of $r$. From the figure we observe that trivial equilibrium point $E_{0}$ is stable for $r \in(0,2.3]$ and DFE $E_{1}$ is stable for $r \in(2.3,2.39)$. Then all three populations exist at the positive state simultaneously and the populations $I$ and $R$ increase with increasing values of $r$ and finally periodic solutions arise in the system for high values of $r(r \geq 3.136)$ (note that for the given parametric values the eigenvalues associated with the Jacobian matrix at $E_{2}(0.95,0.3,0.00013)$ are $-2.31,0 \pm 1.1722 i$ when $r=3.136$, which indicates existence of a periodic solution). From Figures 1 and 6 we can conclude that the system undergoes the supercritical Hopf

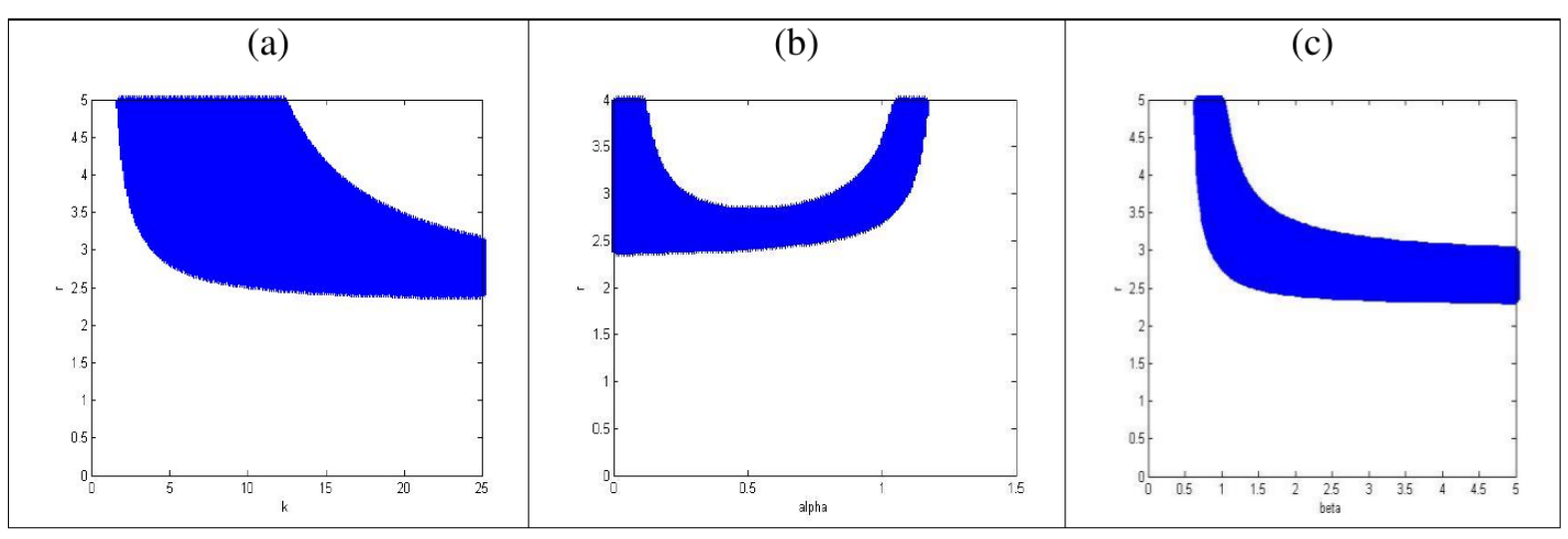

Fig. 5. Stability regions for the endemic equilibrium point $E_{2}\left(S_{2}, I_{2}, R_{2}\right)$ in (a) $(k, r)$ parametric domain and other parametric values are reported in Table 3, (b) $(\alpha, r)$ parametric domain and other parametric values are reported in Table 3, (c) $(\beta, r)$ parametric domain and other parametric values are reported in Table 3

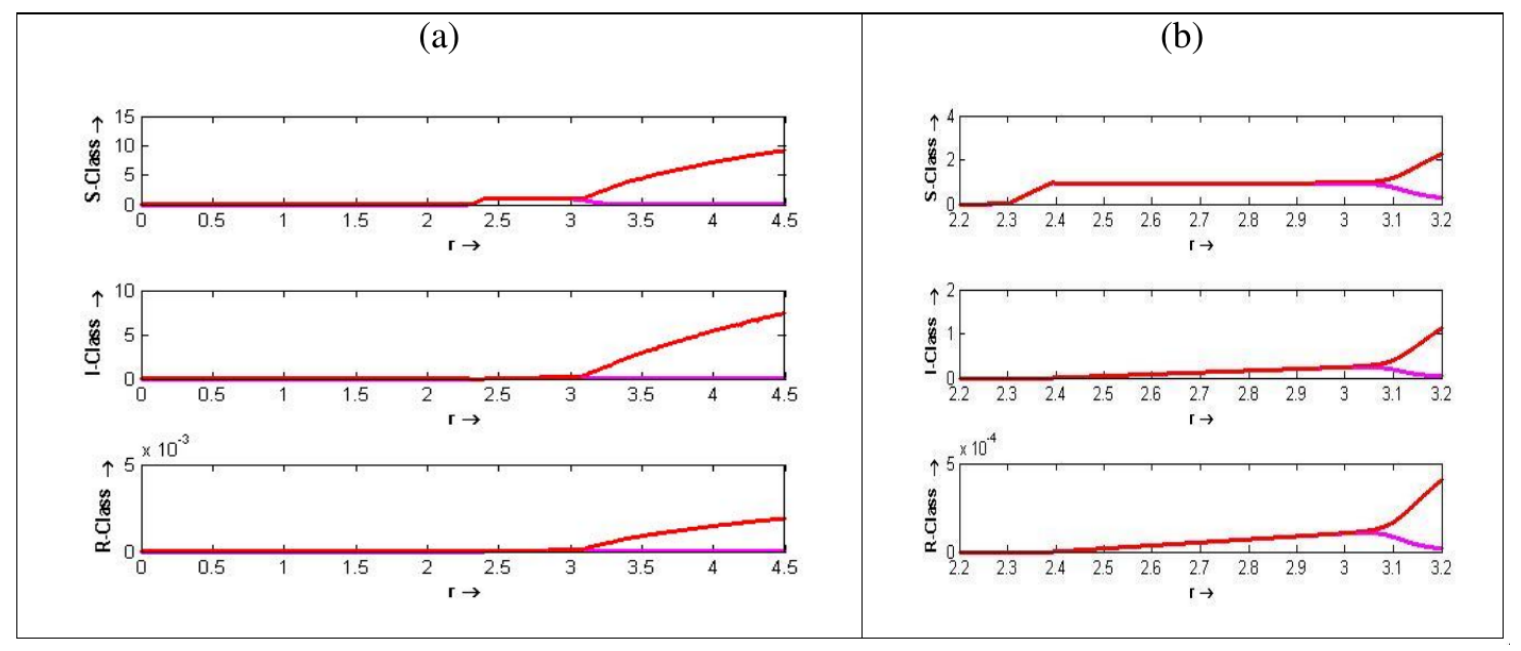

Fig. 6. (a) Bifurcation diagram w.r.t. $r$ and other parametric values reported in Table 3, (b) local amplification of the Figure (a) in the interval $[2.2,3.2]$ 


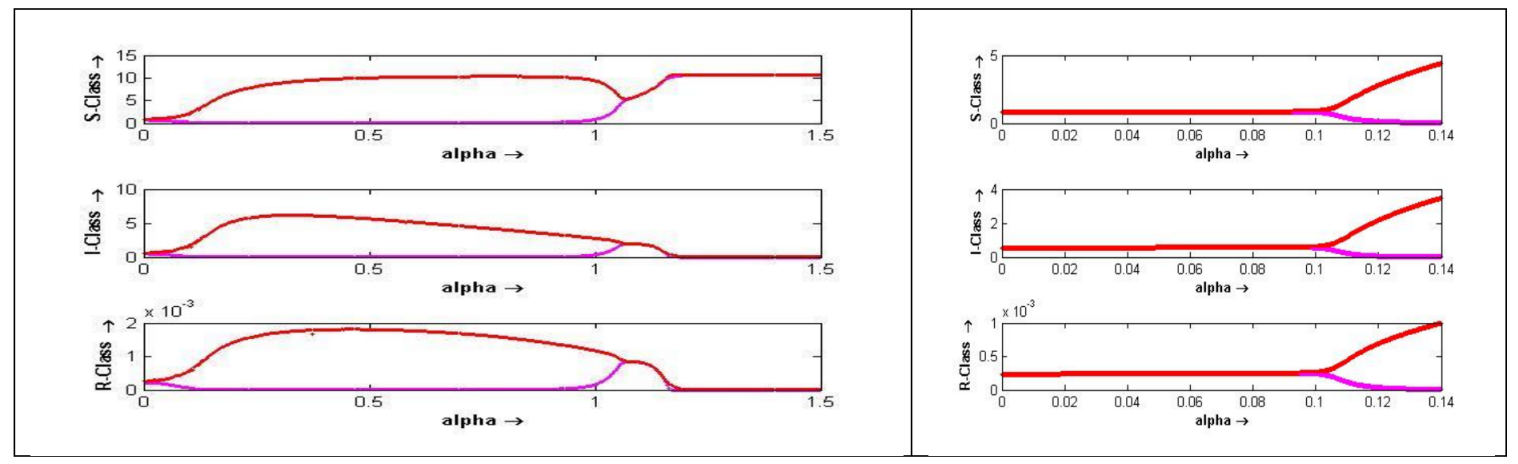

Fig. 7. (a) Bifurcation diagram w.r.t. $\alpha$ and other parametric values reported in Table 3 with $r=4$, (b) local amplification of the Figure (a) in the interval $[0,0.14]$
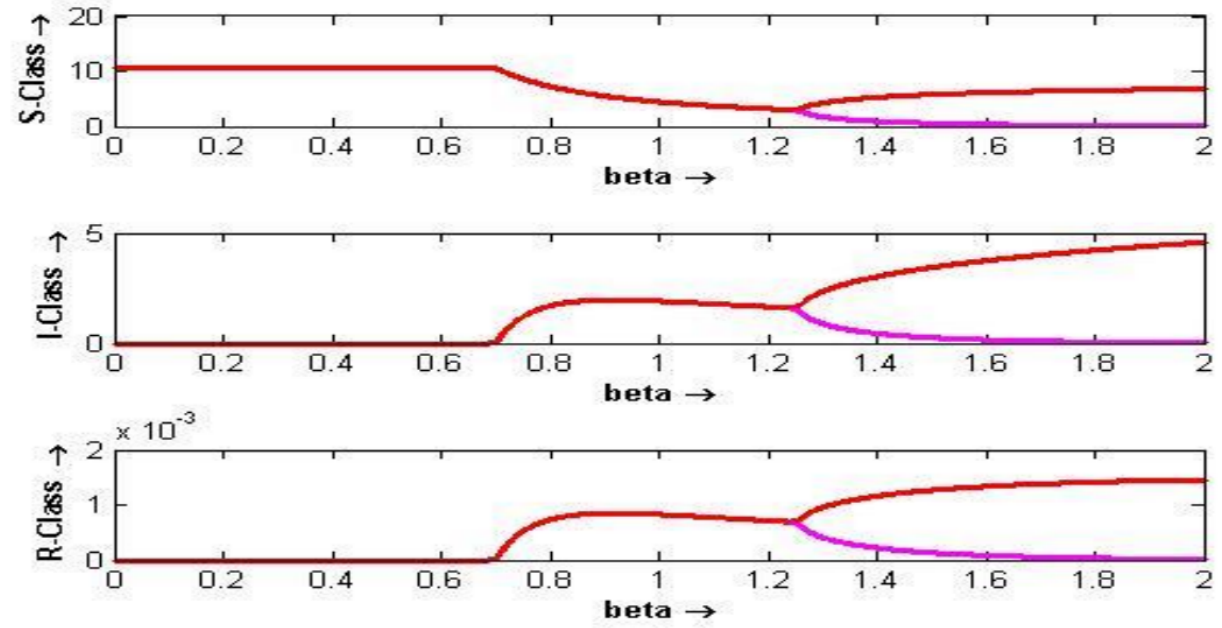

Fig. 8. Bifurcation diagram w.r.t. $\beta$ and other parametric values reported in Table 3 with $r=4$

bifurcation.

To illustrate the effect of inhibitory factor $\alpha$, we plot the bifurcation diagram (cf. Figure 7) by taking $\alpha$ as a bifurcation parameter. This bifurcation diagram shows that the endemic equilibrium point $E_{2}$ is locally stable for lower values of $\alpha$. The system changes from the stable steady state to periodic oscillation at $\alpha=0.1125\left(=\alpha_{1}\right)$ (note that for the given parametric values the eigenvalues associated with the Jacobian matrix at $E_{2}(0.87,0.59,0.00025)$ are $-2.31, \pm 1.8084 i$ when $\alpha=\alpha_{1}$ ) and the solution changes from the periodic solution to the stable steady state at $\alpha=1.053\left(=\alpha_{2}\right)$ (note that for the given parametric values the eigenvalues associated with the Jacobian matrix at $E_{2}(4.82,1.95,0.00084)$ are $-2.31, \pm 0.5927 i$ when $\alpha=\alpha_{2}$ ) and beyond the value of $\alpha_{2}$, no oscillation is observed. This means for $\alpha \in\left(\alpha_{1}, \alpha_{2}\right) E_{2}$ is unstable, and all the populations $S, I, R$ co-exist in the oscillatory mode. Therefore, the system exhibits switching behaviour twice and the system undergoes two Hopf bifurcations as a function of the control parameter $\alpha$. Figure 7 illustrates supercritical and subcritical Hopf bifurcations take place at $\alpha=\alpha_{1}$ and $\alpha=\alpha_{2}$, respectively. The bifurcation diagram also shows that the infected population goes extinct with increasing values of the inhibitory factor. So, the inhibitory coefficient plays a crucial role in the changing of dynamics of the infectious disease.

Next, we have plotted all populations (cf. Figure 8) as the variation of bifurcation parameter $\beta$. It is clear from Figure 8 that there is a range $(0,0.69977)$ of $\beta$ for which the DFE is stable, after that the susceptible population starts to decrease and other two populations increase and finally at $\beta=1.27$ the system experiences the supercritical Hopf bifurcation (note that for the given parametric values the eigenvalues associated with the Jacobian matrix at $E_{2}(2.92,1.57,0.00068)$ are $-2.31,0 \pm 1.325 i$ when $\beta=1.27$ ). Thus, for the low transmission rate of infection the disease is absent but for high transmission rate of infection the disease will persist in the system very strongly and, as it is periodic, the disease is difficult to control.

The role of carrying capacity of the system should not be neglected. The one-parametric bifurcation diagram (cf. Figure 9) demonstrates how the system behaves with the variation of the numerical values of $k$. It suggests that if we increase 

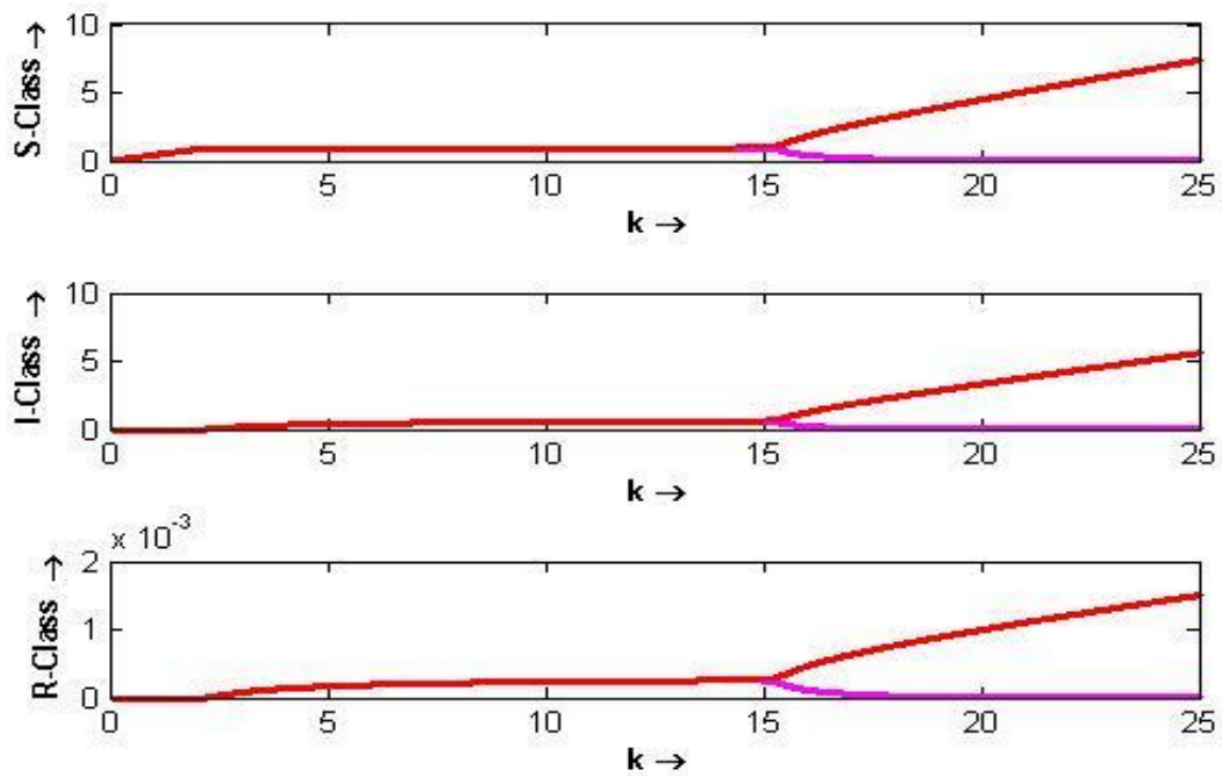

Fig. 9. Bifurcation diagram w.r.t. $k$ and other parametric values reported in Table 3 with $r=4$

the value of $k$, the dynamics of the system moves from stability to oscillation through a point of the supercritical Hopf bifurcation at $k=15.7$ (note that for the given parametric values the eigenvalues associated with the Jacobian matrix at $E_{2}(0.95,0.6,0.00026)$ are $-2.31, \pm 1.67 i$ when $\left.k=15.7\right)$. Periodicity of the solutions for high carrying capacity of the system indicates the disease will come back again in the system.

\section{CONCLUSIONS}

This paper deals with a SIRS model where the logistic growth rate of susceptibles and the inhibitory factor in the incidence rate are considered. The local stability analysis of both hyperbolic and non-hyperbolic equilibrium points is analysed. We have seen that the dynamical system is globally asymptotically stable around the disease free equilibrium point $E_{1}\left(S_{1}, 0,0\right)$ and the endemic equilibrium point $E_{2}\left(S_{2}, I_{2}, R_{2}\right)$ in some domains, which means the solutions converge to the corresponding equilibrium point for all initial values of $S, I$ and $R$ within the specific domain. It is also observed from the obtained result that there exists a threshold value of inhibitory factor $\alpha=\alpha_{0}$, such that the disease free equilibrium point $E_{1}$ is stable for $\alpha>\alpha_{0}$ and is unstable for $\alpha \leq \alpha_{0}$. This implies that the awareness factor plays an important role to control the disease. It was also found that

Tab. 5. Nature of equilibrium points in various ranges of bifurcation parameters, keeping other parameters fixed as in Table 3 with $r=4$

\begin{tabular}{c|c|c|c|c}
\hline Bifurcation parameter & Parameter range & $E_{0}$ & $E_{1}$ & $E_{2}$ \\
\hline$r$ & $0<r \leq 2.3$ & Feasible, stable & Infeasible & Infeasible \\
\hline & $2.3<r<2.39$ & Feasible, unstable & Feasible, stable & Infeasible \\
\hline & $2.39 \leq r<3.136$ & Feasible, unstable & Feasible, unstable & Feasible, stable \\
\hline & $r \geq 3.136$ & Feasible, unstable & Feasible, unstable & Feasible, unstable \\
\hline & $0<\alpha \leq 0.1125$ & Feasible, unstable & Feasible, unstable & Feasible, stable \\
\hline & $0.1125<\alpha \leq 1.053$ & Feasible, unstable & Feasible, unstable & Feasible, unstable \\
\hline & $1.053<\alpha \leq 1.166$ & Feasible, unstable & Feasible, unstable & Feasible, stable \\
\hline & $\alpha>1.166$ & Feasible, unstable & Feasible, stable & Infeasible \\
\hline & $0<\beta<0.69977$ & Feasible, unstable & Feasible, stable & Infeasible \\
\hline & $0.69977 \leq \beta<1.27$ & Feasible, unstable & Feasible, unstable & Feasible, stable \\
\hline & $\beta \geq 1.27$ & Feasible, unstable & Feasible, unstable & Feasible, unstable \\
\hline & $0<k<2.2402$ & Feasible, unstable & Feasible, stable & Infeasible \\
\hline & $2.2402 \leq k<15.7$ & Feasible, unstable & Feasible, unstable & Feasible, stable \\
\hline & $k \geq 15.7$ & Feasible, unstable & Feasible, unstable & Feasible, unstable \\
\hline
\end{tabular}


the system undergoes a Hopf bifurcation leading to a family of periodic solutions that bifurcates from $E_{2}$ for suitable values of the intrinsic growth rate of susceptible class. We have proved that there is no closed orbit in $D_{2}$ for the system (1) and the reduced system for $\mu=0$ does not have any closed orbit if $k \alpha<1$. It is also proved analytically that the trivial and disease free equilibrium points are unstable when endemic equilibrium point exists.

Finally, in section 6 we have drawn the stability or instability regions for the disease free and endemic equilibrium points. The bifurcation diagram is a very useful tool to describe the stability analysis and long-term behaviour of the system in a single figure. Therefore, we have plotted the bifurcation diagrams with respect to the parameters $r, \alpha, \beta$ and $k$. A detailed presentation of the relationship between the bifurcation parameters $r, \alpha, \beta, k$ and the nature of the equilibrium points is done in section 6 and we have summarized this in Table 5 . The entire study of the paper is mainly based on the deterministic framework and our proposed model is valid for a large population only. The work is a theoretical modelling and it can be further justified using experimental results.

\section{Acknowledgement}

We are very grateful to Dr. Malay Banerjee, Associate Professor, Department of Mathematics and Statistics, IIT, Kanpur for his careful reading, valuable comments and helpful suggestions, which have helped us to improve the presentation of this work significantly. The authors are also grateful to the anonymous referee for careful checking of the details and for helpful comments that have improved this paper.

\section{References}

[1] W.Kermack, A. Mckendric, A contribution to mathematical theory of epidemics, Proc. Roy. Soc. Lond. A Mat. 115, 700-721 (1927).

[2] W.Kermack, A. Mckendric, Contributions to the mathematical theory of epidemics-I, Bulletin of Mathematical Biology 53, 33-55 (1991).

[3] O. Diekman, J.A.P. Heesterbeek, Mathematical Epidemiology of Infectious Disease, Wiley, New York, 2000.

[4] N.T.J. Bailey, The Mathematical Theory of Infectious Diseases, Griffin, London, 1975.

[5] J.D. Murray, Mathematical Biology, Springer, New York, 1993.

[6] R.M. Anderson, R.M. May, Infectious Diseases of Humans: Dynamics and Control, Oxford University Press, 1998.

[7] Z. Ma, J. Li (eds.), Dynamical Modelling and Analysis of Epidemics, World Scientific, 2009.

[8] F. Brauer, C. Castillo-Chavez, Mathematical Models in Population Biology and Epidemiology, Springer, 2011.

[9] Y. Enatsu, E. Messina, Y. Muroya, Y. Nakata, E. Russo and A. Vecchio, Stability analysis of delayed SIR epidemic models with a class of nonlinear incidence rates, Applied Mathematics and Computation 218, 5327-5336 (2012).
[10] J.J. Wang, J.Z. Zhang, Z. Jin, Analysis of an SIR model with bilinear incidence rate, Nonlinear Anal. RWA 11, 2390-2402 (2009).

[11] J. Wang, S. Liu, B. Zhen and Y. Takeuchi, Qualitative and bifurcation analysis using an SIR model with a saturated treatment function, Mathematical and Computer Modelling 55, 710-722 (2012).

[12] T.K. Kar, P. Mandal, Global dynamics and bifurcation in delayed SIR epidemic model, Nonlinear analysis: Real World Applications 12, 2058-2068 (2011).

[13] W.M. Liu, S.A. Levin, Y. Iwasa, Influence of nonlinear incidence rates upon the behaviour of SIRS epidemiological models, J. Math. Biol 23, 187-204 (1986).

[14] J.Z. Zhang, Z. Jin, Q.X. Liu, Z.Y. Zhang, Analysis of a delayed SIR model with non-linear incidence rate, Discrete Dynamics in Nature and Society, (2008).

[15] L. Cai, S. Guo, X. Li, M. Ghosh, Global dynamics of a dengue epidemic mathematical model, Chaos Solitons Fractals 42, 22972304 (2009).

[16] Z.X. Liu, S. Liu, H. Wang, Backward bifurcation of an epidemic model with standard incidence rate and treatment rate, Nonlinear Anal. RWA 348, 433-443 (2008).

[17] L. Zhou, M. Fan, Dynamics of an SIR epidemic model with limited medical resources revisited, Nonlinear Anal. RWA 13, 312-324 (2012).

[18] D. Xiao, S. Ruan, Global analysis of an epidemic model with non-monotone incidence rate, Math. Biosci. 208, 419-429 (2007).

[19] X. Zhang, X.N. Liu, Backward bifurcation and global dynamics of an SIS epidemic model with general incidence rate and treatment, Nonlinear Anal. RWA 10, 565-575 (2009).

[20] V. Capasso, G. Serio, A generalization of the KermackMckendrick deterministic epidemic model, Math. Biosci. 42, 43-61 (1978).

[21] P. Van den Driessche and J. Watmough, Reproduction Numbers and Sub-Threshold Endemic Equilibria for Compartmental Models of Disease Trans-mission, Mathematical Biosciences 180, 29-48 (2002).

[22] J.K. Hale, Ordinary Differential Equations $2^{\text {nd }}$ ed., Krieger, Basel, 1980.

[23] L. Perko, Differential Equations and Dynamical Systems, Springer, New York, 2000.

[24] T.C.Gard, Persistence in Food Webs: Holling-Type Food Chains, Math Biosci 49, 61-67 (1980).

[25] M.B. Trawicki, Deterministic Seirs Epidemic Model for Modeling Vital Dynamics, Vaccinations, and Temporary Immunity, Mathematics 5(1), 7 (2017).

[26] L. Wang, D. Zhou, Z. Liu, D. Xu, X. Zhang, Media alert in an SIS epidemic model with logistic growth, Journal of Biological Dynamics 11, 120-137 (2017).

\section{Appendix: Boundedness and Permanence of the System}

The concept of boundedness and permanence was introduced in population biology and has been studied extensively. This concept is very important in mathematical epidemiology as well. Boundedness of a system implies that the system is biologically well behaved and permanence implies that the disease will be maintained globally, irrespective of the initial composition. 
Theorem A. The region

$$
D=\left\{(S, I, R) \in R_{+}^{3}: S+I+R \leq \frac{r k}{d}\right\}
$$

is a positively invariant set for the system (1).

Proof. Let $N=S+I+R$.

Then

$$
\begin{gathered}
\frac{d N}{d t}=r S\left(1-\frac{S}{k}\right)-d N= \\
-\frac{r}{k}(k-S)^{2}-d N+r k-r S \leq r k-d N .
\end{gathered}
$$

It follows that $\lim \sup N(t) \leq \frac{r k}{d}$ as $t \rightarrow \infty$.

Hence the theorem is proved.

Theorem B. If $r>d+\gamma$, the system (1) is persistent and permanent.

Proof. We make use of the function $\rho(S, I, R)=S^{r_{1}} I^{r_{2}} R^{r_{3}}$, where $r_{i}$ 's are positive constants to be determined in the process of verifying that $\rho$ is a persistence function for the system (1).

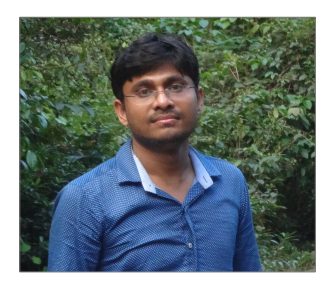

Jayanta Kumar Ghosh is a Research Scholar in the Department of Applied Mathematics, University of Calcutta. His research field includes Mathematical Epidemiology.

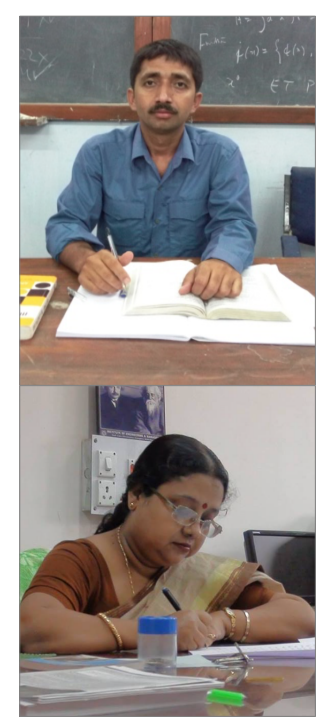

Uttam Ghosh is Assistant Professor of Applied Mathematics at the University of Calcutta. His research field includes Fractal geometry, Information theory, Percolation theory, Biomathematics and Fractional Calculus. He has 35 publications in reputed national and international journals.

Susmita Sarkar is Professor of Applied Mathematics, University of Calcutta. Her field of research includes Plasma Dynamics, Biomathematics and Fractional Calculus. She has more than 62 research publications in reputed international journals. Dr. Susmita Sarkar was TWAS Associate and ICTP regular Associate. 